\title{
Microchip Immunoassay Using High Density Bioconjugation on the Phospholipid Polymer Interface
}

\author{
Kazuki Nishizawa, Tomohiro Konno, Madoka Takai and Kazuhiko Ishihara \\ Department of Materials Engineering and Center for NanoBio Integration, The University of Tokyo, \\ 7-3-1, Hongo, Bunkyo-ku, Tokyo, 113 8656, Japan \\ Fax: 81-3-5841-8647, e-mail: nishizawa@mpc.t.u-tokyo.ac.jp
}

\begin{abstract}
We developed a new solid biointerface for highly sensitive microchip immunoassay by integrating a phospholipid polymer (poly [2-methacryloyloxyethyl phosphorylcholine (MPC) -co- $n$-butyl methacrylate (BMA) -co- $p$-nitrophenyloxycarbonyl poly(ethylene glycol) methacrylate (MEONP)]: PMBN) with a nanoscale surface modification process known as the electrospray deposition (ESD) method. The PMBN surface which can conjugate antibodies prevented the nonspecific protein adsorption. The PMBN surface coated by ESD has a nanosphere-shaped structure and showed a high water repellent property. A large amount of antibodies were immobilized using the PMBN surface coated by ESD with surfactant in the solution of primary antibodies. When surface tension of aqueous solution of antibodies was lowered, antibodies could bind to inner surface of PMBN nanostructure and arranged three-dimensionally. High density antibodies immobilized PMBN surface coated by ESD enhanced the specific signal and showed low nonspecific signal in the microchip immunoassay. The PMBN surface coated by ESD realized highly sensitive microchip immunoassay for detecting a small volume of sample in a short time. Key words: MPC polymer, biointerface, immunoassay, biosensor, electrospray deposition
\end{abstract}

\section{INTRODUCTION}

Recently, miniaturized biosensors for microdiagnostic devices have been designed to allow bedside monitoring of chemicals and biomolecules $[1,2]$. Enzyme-linked immunosorbent assay (ELISA) has been the most widely used method $[3,4]$ in the fields of biochemistry and clinical diagnosis. A microchip-based ELISA system is a promising interdisciplinary tool for the next generation clinical diagnosis [5]. Compared with the conventional ELISA carried out in a polystyrene microtiter plate, the microchip-based ELISA enhances the reaction efficiency. In addition, it has other advantages such as a short assay time, low sample consumption, and assay automation [6]. However, the significant decrease in sample volume results in low specific signals. Furthermore, in the microscale environment, the high surface area/volume ratio magnifies the influence of nonspecific binding of an analyte or a labeled antibody to the solid surface. These factors are the cause of low specific signal and high noise level. To develop a highly sensitive microchip-based ELISA system, both enhancement of specific signals and reduction of nonspecific signals should be achieved.

To reduce the nonspecific signal, protein-based blocking reagents such as bovine serum albumin (BSA), and casein are commonly used in laboratories worldwide. However, protein-based blocking reagents denature easily and cross-react between detection reagents and blocking reagents persist as one of the main causes of a high background and low signal-to-noise ratio. Based on the concept of protein-free blocking reagent, several types of artificial blocking reagents such as Tween20 [7] and poly(ethylene glycol) [8] have been studied and reported. The 2-methacryloyloxyethyl phosphorylcholine (MPC) polymer, which is based on the structure of cell membranes with phosphorylcholine groups, is reported as an excellent blocking reagent for ELISA [9]. It shows a high resistivity to protein adsorption and effectively decreases the denaturation of biomolecules [10].

To enhance the specific signal, it is important to immobilize a large amount of antibodies on a solid surface with its activity high. Microbeads-based assay [11] is beneficial for increasing the amount of immobilized antibodies. It was reported that when the primary antibody is supported by some ligand via a spacer, the reaction efficiency is enhanced due to the high mobility of the antibody [12].

In this study, we developed a solid biointerface for highly sensitive microchip ELISA by integrating a MPC polymer, namely, poly [MPC - co- $n$-butyl methacrylate (BMA) -co- $p$-nitrophenyloxycarbonyl poly(ethylene glycol) methacrylate (MEONP)]: PMBN (Fig. 1) with a nanoscale surface modification process known as the electrospray deposition (ESD) method [13]. The MEONP unit contains active ester groups for the conjugation of antibodies via the oxyethylene chain. The PMBN comprises units to prevent nonspecific adsorption as well as to conjugate antibodies in a single polymer chain. Our previous study reported that the PMBN surface reduced the nonspecific signal and maintained a residual activity of immobilized antibodies after long-term storage [14].

ESD method can produce nano-microscaled structure films of polymers, proteins, and DNA in forms ranging from spheres to fibers. Furthermore, ESD can also apply to microchips on which a conductive substrate has been formed by micro patterning. We sprayed PMBN using 
ESD method and carried out ELISA in a microtiter plate, and this surface property was discussed [15]. In this report, we applied the PMBN nanostructured surface coated by ESD to the microchip ELISA, and the amount of immobilized antibodies, specific and nonspecific signals were investigated.

\section{EXPERIMENTAL}

2.1 Synthesis of bioconjugated phospholipid polymer

PMBN, the phospholipid polymer for bioconjugation, was prepared using MPC, BMA, and MEONP. MPC and MEONP were synthesized as described previously in detail $[16,17]$. BMA was purchased from Nacalai Tesque, Inc. (Kyoto, Japan). PMBN was synthesized by conventional radical polymerization of MPC, BMA, and MEONP by using $\alpha, \alpha$ '-azobisisobutyronitrile (AIBN) as an initiator. After polymerization, the reaction mixture was precipitated using a mixture of chloroform and diethylether $(2: 8)$ as a solvent. The ratio of monomer unit composition in PMBN was determined by ${ }^{1} \mathrm{H}-\mathrm{NMR}$ (JEOL JNM-NR30, Tokyo, Japan). The molecular weight of PMBN was evaluated by gel-permeation chromatography (GPC) (OHpak SB-804 HQ column, Shodex, Tokyo, Japan) with poly(ethylene oxide) (PEO) standards in a mixture of water and methanol (3:7).

\subsection{Preparation of nanostructured polymer surface}

To make conductive substrate for ESD, $\mathrm{Au}$ was sputtered on a slide glass (Matsunami Glass Ind., Ltd., Osaka, Japan) using sputtering device (SCOTT-C3, Ulvac Kiko Inc., Kanagawa, Japan). 5 wt $\%$ ethanol solution of PMBN was sprayed to the Au surface by the ESD device (esprayer ES-1000, fuence, Tokyo, Japan) at a voltage of $20 \mathrm{kV}$. To compare with ESD sprayed surface, another $\mathrm{Au}$ sputtered glass was dipped with a $0.2 \mathrm{wt} \%$ ethanol solution of PMBN and put in the same solvent vapor atmosphere for one night and then dried under vacuum overnight. The morphologies of the surfaces were observed using a scanning electron

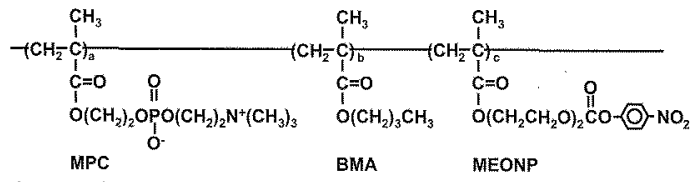

Fig. 1 Chemical structure of PMBN.

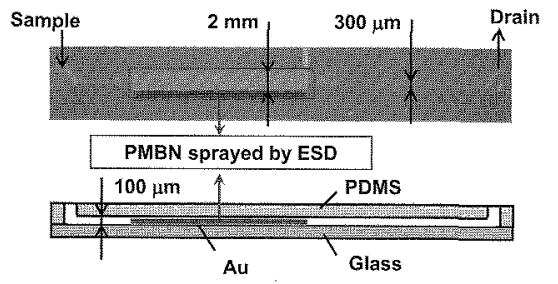

Fig. 2 Photograph and schematic representation of microchip for ELISA.

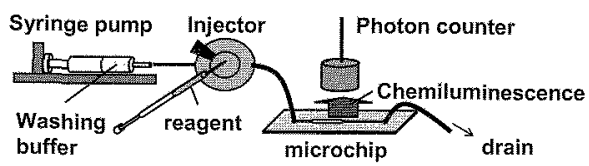

Fig. 3 Schematic representation of microchip ELISA system. microscope (SM-200 scanning microscope, Topcon Co, Tokyo, Japan) at an acceleration voltage of $15 \mathrm{keV}$. The static contact angles of the water droplets were measured by the sessile drop method using a goniometer (Kyowa Interface Science, Tokyo, Japan) at room temperature.

2.3 Quantification of amount of immobilized antibodies PMBN ESD coated, dip coated and non-coated Au sputtered cover glasses (13 $\mathrm{mm} \phi$, Matsunami Glass Ind., Ltd., Osaka, Japan) were immersed in $1 \mathrm{~mL}$ of $10 \mu \mathrm{g} / \mathrm{mL}$ mouse IgG (Sigma-Aldrich, Corp., St. Louis, USA) phosphate buffer solution ( $\mathrm{pH} 8$ ). To lower the surface tension of the IgG solution, Tween20 (Sigma-Aldrich, Corp., St. Louis, USA) was added in the IgG solution from 0 to $1 \mathrm{wt} \%$. After incubation for $10 \mathrm{~h}$ at $25^{\circ} \mathrm{C}$, a protein analysis kit (Micro BCA ${ }^{\mathrm{TM}}$ protein assay reagent kit, Pierce, Rockford, IL, USA) based on the bicinchoninic acid (BCA) method was used to determine the concentration of the mouse $\operatorname{IgG}$ in the supernatant solution on these plates. Absorbance at $560 \mathrm{~nm}$ was measured using a multilabel counter (Wallac ARVOsx1420, Perkin Elmer). The amount of immobilized mouse $\operatorname{Ig} G$ on these plates were calculated by subtracting initial concentration of the mouse $\operatorname{IgG}(10$ $\mu \mathrm{g} / \mathrm{mL}$ ) from the concentration of the mouse $\operatorname{IgG}$ in the supernatant solution.

\subsection{Microchip fabrication}

The patterned master was made from SU-8 (MicroChem. Corp., Newton, MA, USA) resist on a slide glass by photolithography. The precursor of polydimethylsiloxane (PDMS) and cross-linker (Silpot 184, Toray-Dow Corning Co., Tokyo, Japan) were fully mixed at the ratio of 10:1 by mass. The mixture was poured over the patterned master and degassed for $1 \mathrm{~h}$ at room temperature. The curing reaction was then carried out at $60^{\circ} \mathrm{C}$ for $6 \mathrm{~h}$. After removing the PDMS replica, the holes for sample inlet and outlet were made by drilling. The depth of microchannel was $100 \mu \mathrm{m}$ and the width is $300 \mu \mathrm{m}$ and $2 \mathrm{~mm}$ for channel and reaction area, respectively (Fig. 2). Au patterned slide glass was made by sputtering using stainless steel mask, and oxygen plasma treatment $(300 \mathrm{~W}, 1 \mathrm{~min}$ ) was carried out using plasma reactor (PR500, Yamato Scientific Co., Ltd., Tokyo, Japan), and then PMBN was sprayed by ESD on the $2 \mathrm{~mm} \times 2 \mathrm{~cm}$ Au area. Then, the PDMS was stuck on the PMBN ESD coated slide glass after oxygen plasma treatment $(85 \mathrm{~W}, 10 \mathrm{sec})$.

\subsection{Protocol of microchip ELISA}

ELISA was carried out in the microchip with PMBN coated surfaces as following protocol. $5 \mu \mathrm{L}$ of $20 \mu \mathrm{g} / \mathrm{mL}$ the anti-[human Thyroid Stimulating Hormone (TSH)] mouse IgG (Bioclone Australia Pty Ltd., Sydney, Australia) in phosphate buffer solution (pH 8) containing the $0-1 \mathrm{wt} \%$ of Tween 20 was pipetted into the microchannel and allowed to react with the active ester of PMBN for $5 \mathrm{~min}$. After incubation, the microchannel was washed with $50 \mu \mathrm{L}$ of phosphate buffered saline (PBS, pH 7.1). To reduce the nonspecific binding of proteins on the upper PDMS side of microchannel, poly(MPC-co-BMA) (PMB30W) [18] was used as a blocking reagent. To compare with conventional surface for ELISA, bovine serum albumin 
(BSA) (Sigma-Aldrich, Corp., St. Louis, USA) was also used as a blocking reagent for non-coated Au surface. $5 \mu \mathrm{L}$ of $1 \mathrm{wt} \%$ of PMB30W or $1 \mathrm{wt} \%$ of BSA in PBS solution was pipetted into the microchannel. After incubation for $3 \mathrm{~min}$, the microchannel was washed with $50 \mu \mathrm{L}$ of PBS solution. To perform the antigen-antibody reaction, $5 \mu \mathrm{L}$ of $0-10 \mu \mathrm{IU} / \mathrm{mL}$ human TSH (Biogenesis Ltd., England, UK) in PBS solution was pipetted into the microchannel. After incubation for $5 \mathrm{~min}$, the microchannel was washed with $100 \mu \mathrm{L}$ of PBS solution containing $0.1 \mathrm{wt} \%$ Tween 20 , and then 1 $\mu \mathrm{g} / \mathrm{mL}$ horseradish peroxidase (HRP) labeled hTSH IgG (Leinco Technologies, Inc., St. Louis, USA) in PBS solution containing $1 \mathrm{wt} \%$ BSA was pipetted into the microchannel. After incubation for $5 \mathrm{~min}$, the wells were washed with $100 \mu \mathrm{L}$ of PBS solution containing $0.1 \mathrm{wt} \%$ Tween20, and then microchip was connected with polyetheretherketone (PEEK) tube and set in the spectrometer (FP-6500, JASCO Corp., Tokyo, Japan). Washing buffer (PBS solution containing $0.1 \mathrm{wt} \%$ Tween20) was flowed at $5 \mu \mathrm{L} / \mathrm{min}$ using micro syringe pump (ESP-32, Eicom Corp., Kyoto, Japan). Then, 20 $\mu \mathrm{L}$ of chemiluminescence substrate for HRP (Femtoglow Plus HRP Substrate; Michigan Diagnostics. Troy, MI, USA) was injected form a sample injector (7725, Rheodyne LLC, CA, USA) at $5 \mu \mathrm{L} / \mathrm{min}$. The chemiluminescence intensity from the microchip was measured. Schematic representation of microchip ELISA system is shown in Fig. 3.

\section{RESULTS AND DISCUSSION}

3.1 Morphology and wettability of PMBN surfaces

PMBN was synthesized, and the monomer unit compositions were MPC: BMA: MEONP $=20: 60: 20$ in feed and 22:65:13 in copolymer determined by ${ }^{1} \mathrm{H}-\mathrm{NMR}$. The weight average molecular weight was $3.0 \times 10^{4}$ determined by GPC.

Fig. 4 shows the SEM images of surfaces sprayed with a PMBN by the ESD device and dip-coated with a PMBN. A surface dip-coated with PMBN is very smooth, while the nanosphere-shaped polymer network was observed on the surface sprayed by ESD.

Fig. 5 shows the shapes of water droplets and contact angles. PMBN surface sprayed by ESD shows much larger contact angle than that of dip coated surface. This indicates that high roughness and porosity of ESD coated surface caused its water-repellent property. There are two well-established models for wetting on rough surfaces: Wenzel [19] and Cassie-Baxter [20] equations. Wetting behaviors of ESD coated highly uneven surfaces can be explained by these two well-known models [21]. In the case of water droplet including surfactant $(0.1 \%$, and $1 \%$ Tween 20 aqueous solution), the contact angles on the PMBN surface coated by ESD was decreased in proportion to the concentration of Tween20.

3.2 Effect of surfactant on amount of immobilized antibodies

Amount of immobilized antibodies on the PMBN surface was evaluated using micro BCA method. As shown in Fig. 6, Tween 20 in the solution of antibodies as surfactant enhanced the amount of immobilized antibodies on the PMBN nanostructured surfaces coated by ESD method. In proportion to the concentration of Tween 20 in the solution of antibodies, the amount of immobilized antibodies on the PMBN surface coated by ESD was increased. This result indicates that the aqueous solution of antibodies can hardly access to inner surface of nanostructured PMBN coated by ESD because of its water-repellent property. However, antibodies in the aqueous solution with surfactant can permeate and bind to the inner surface of nanostructured PMBN coated by ESD due to the surface tension of aqueous solution of antibodies is reduced by surfactant. In the case of using $1 \%$ Tween 20 in the solution of antibodies, the amount of immobilized antibodies was very high, $3.4 \mu \mathrm{g} / \mathrm{cm}^{2}$. If antibodies are immobilized on two-dimensional flat surface with closest packing in end-on position, the amount of immobilized antibodies is calculated as $0.65 \mu \mathrm{g} / \mathrm{cm}^{2}$ (the shape of antibody is supposed to be lens-shaped spheroid which size is 15 $\mathrm{nm} \times 15 \mathrm{~nm} \times 3 \mathrm{~nm}$ ). Considering this calculation, 3.4 $\mu \mathrm{g} / \mathrm{cm}^{2}$ of immobilized antibodies are arranged three-dimensionally on the nanostructured PMBN surface.

3.3 Chemiluminescence detection in microchip ELISA

ELISA using microchip with PMBN coated surfaces was carried out to evaluate the specific and nonspecific signals. The results were shown in Fig. 7. In the case of non-coated $\mathrm{Au}$ surface using BSA as a blocking reagent, specific signal (TSH $=10 \mu \mathrm{IU} / \mathrm{mL}$ ) was relatively high, but nonspecific signal (TSH $=0 \mu \mathrm{IU} / \mathrm{mL}$ ) was also high. This indicates that the blocking effect of BSA is not sufficient and it can not fully restraint nonspecific binding of HRP labeled IgG on the surface. On the other hand, PMBN ESD coated surface showed much lower nonspecific signal than that of $\mathrm{Au}$ surface using BSA. It is considered that MPC unit in the PMBN prevented the nonspecific adsorption of HRP labeled IgG effectively. With regard to specific signals, ESD coated surface without Tween 20 was low, but ESD coated surface with $1 \%$ Tween20 in solution of primary antibodies considerably higher. Because specific signals have correlation with the amount of immobilized antibodies,
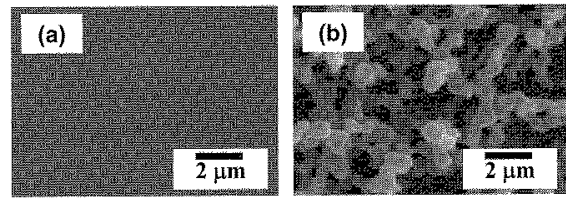

Fig. 4 Scanning electron microscope images of the PMBN surfaces (a) dip coating and (b) ESD coating.
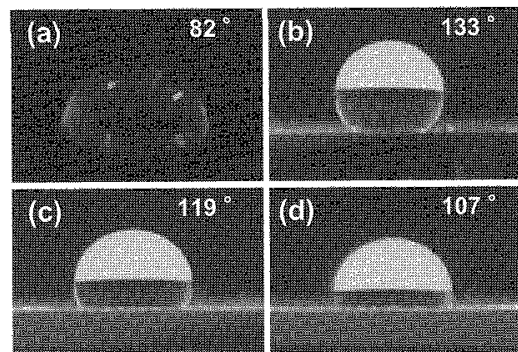

Fig. 5 Photographs of water droplets and contact angles on the PMBN surfaces (a) dip coating, (b) ESD coating, (c) $0.1 \%$ Tween 20 in water on ESD coating and (d) $1 \%$ Tween 20 in water on ESD coating. 


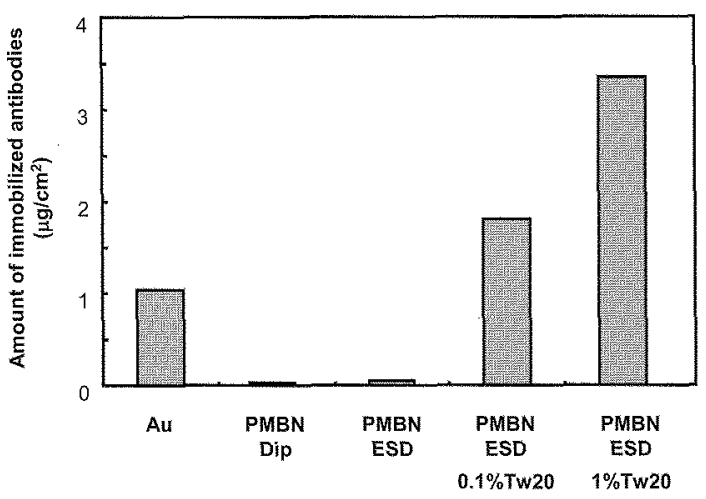

Fig. 6 Amount of immobilized antibodies obtained by micro BCA results. Tw20 indicates Tween 20 in the solution of antibodies.

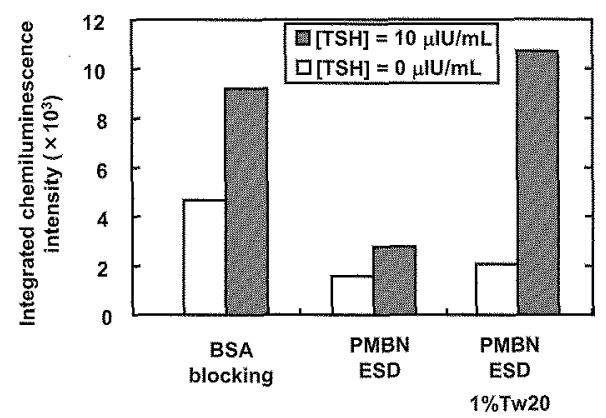

Fig. 7 Integrated chemiluminescence intensity obtained by microchip ELISA. Tw20 indicates Tween 20 in the solution of antibodies.

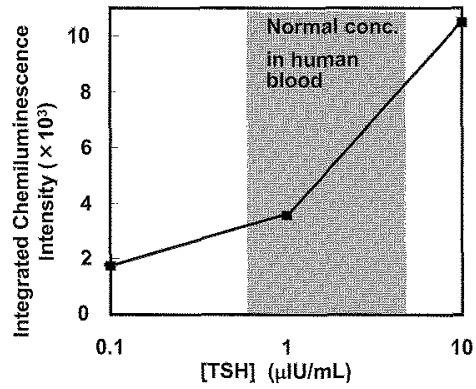

Fig. 8 Standard curve of TSH obtained by microchip ELISA using PMBN surface coated by ESD with $1 \%$ Tween 20 in the solution of antibodies.

the main factor of high specific signal in the case of PMBN ESD coated surface with Tween20 was large amount of immobilized antibodies on this surface. Both enhancement of specific signal and reduction of nonspecific signal were achieved in the case of PMBN ESD coated surface with Tween20, the sensitivity was enhanced to 2.6 times as much as Au surface with BSA. Using PMBN ESD coated surface with Tween20, standard curve of TSH was obtained and showed good linearity in human blood normal concentration (Fig. 8).

Comparing with conventional ELISA using microtiter plate, assay time on the microchip was shortened to 20 min from about $4 \mathrm{~h}$ and sample consumption was reduced to $5 \mu \mathrm{l}$ from about $100 \mu \mathrm{L}$.

\section{CONCLUSION}

The bioconjugated phospholipid polymer, PMBN, was synthesized and nanosphere-shaped PMBN surface was constructed by using ESD method. The PMBN surface coated by ESD showed a high water repellent property because of its high surface roughness. A large amount of antibodies were immobilized using the PMBN surface coated by ESD with $1 \%$ Tween 20 in the solution of primary antibodies. When surface tension of aqueous solution of antibodies was lowered by Tween 20 , antibodies could bind to inner surface of PMBN nanostructure and arranged three-dimensionally. High density antibodies immobilized PMBN surface coated by ESD enhanced the specific signal and showed low nonspecific signal in the microchip immunoassay. The nanosphere-shaped PMBN surface coated by ESD realized highly sensitive microchip ELISA for detecting a small volume of sample $(5 \mu \mathrm{L})$ in a short time $(20$ min).

\section{References}

[1] D. R. Reyes, D. Iossifidis, P. A. Auroux, A. Manz, Anal. Chem. 74, 2623 (2002).

[2] P. A. Auroux, D. Iossifidis, D. R. Reyes, A. Manz, Anal. Chem. 74, 2637 (2002).

[3] A. G. Farr, P. K. Nakane, J. Immunol. Method. 47, 129 (1981).

[4] L. Orci, Diabetologia 28, 528 (1985).

[5] P. Arenkov, A. Kukhtin, A. Germmell, S.

Voloshchuk, V. Chupeeva, A. Mirzabekov, Anal. Biochem. 278, 123 (2000).

[6] J. S. Rossier, H. H. Girault, Lab Chip 1, 153 (2001).

[7] E. Engvall, P. Perlmann, J. Immunol. 109, 129-135 (1972).

[8] Y. Nagasaki, H. Kobayashi, Y. Katsuyama, T. Jomura, T. Sakura, J. Colloid Interface Sci. 309, 524-530 (2007).

[9] S. Sakaki, Y. Iwasaki, N. Nakabayashi, K. Ishihara, Polym. J. 32, 637 (2000).

[10] K. Ishihara, H. Nomura, T. Mihara, K. Kurita, Y. Iwasaki, N. Nakabayashi, J. Biomed. Mater. Res. 39, 323 (1998).

[11] S. Kiichi, M. Yamanaka, T. Hagino, $M$. Tokeshi, H. Kimura, T. Kitamori, Lab. Chip 4, $570(2004)$.

[12] B. C. Weimer, M. K. Walsh, X. Wang, J. Biochem. Biophys. Meth. 45, 211-219 (2000).

[13] J. Doshi, J, Reneker, D. H. Reneker, J.

Electrostat. 35, 151 (1996).

[14] K. Nishizawa, T. Konno, M. Takai, K. Ishihara, Biomacromolecules in press.

[15] K. Nishizawa, T. Konno, M. Takai, K.

Ishihara, Trans. Mater. Res. Soc. Jpn., 32(2), 587 (2007).

[16] K. Ishihara, T. Ueda, N. Nakabayashi, Polym. J. 22, 355 (1990).

[17] T. Konno, J. Watanabe, K. Ishihara, Biomacromolecules 5, 342 (2004).

[18] K. Ishihara, Y. Iwasaki, N. Nakabayashi, Polym. J. 31, 1231 (1999).

[19] R. N. Wenzel, Ind. Eng. Chem. 28, 988 (1936).

[20] A. B. D. Cassie, S. Baxter, Trans. Faraday Soc. 40 , 546 (1944).

[21] T. Mizukoshi, H. Matsumoto, M. Minagawa, A. Tanioka, J. Appl. Polym. Sci. 103, 3811 (2007). 